\title{
Una minoría entre las minorías: Estudios Judíos en el Perú
}

A minority among minorities: Jewish Studies in Peru

Romina Yalonetzky yalonetzky.r@pucp.pe

Pontificia Universidad Católica de Perú

Perú

\section{Resumen}

El artículo explora la situación de los Estudios Judíos sobre el Perú y desde el Perú. A través de una revisión de los catálogos bibliográficos de la Biblioteca Nacional del Perú y de los de seis de las universidades más prestigiosas del país, se ofrece una mirada al inventario de producción académica y no académica sobre lo judío peruano disponible en la actualidad. Sobre la base de las características de dicha producción, esto es, temas abordados, tipo de documento, tipo de edición, circunstancias de publicación; y partiendo de su propia experiencia como investigadora de lo judío en el Perú, la autora ofrece una reflexión sobre las condiciones para el desarrollo de investigaciones en torno a lo peruano-judío.

Palabras clave: Perú, Estudios Judíos, minorías peruanas, Estudios étnicos, religión.

\begin{abstract}
The article explores the situation of Jewish Studies about Peru and from Peru. An overview of the available inventory of academic and non-academic writings on Jewish


Peru is presented after having reviewed the library catalogues of six of the most prestigious universities in the country and the catalogue of Peru's National Library. Based on the type of work, that is, topics addressed, type of document, editing, context of publication, and based on her own experience as a researcher of Jewishness in Peru, the author offers a reflection on the conditions for research on Peruvian-Jewishness.

Keywords: Peru - Jewish Studies - Peruvian minorities - Ethnic Studies - religion.

\section{Introducción}

¿Hay algo así como Estudios Judíos en el Perú? De haberlos, ¿qué es eso que los define? ¿Será que incluyen al conjunto de productos académicos que mantengan relación o hagan referencia a algo judío en el Perú, elaborados en el país? ¿O deberían reducirse a su institucionalización por medio de la creación de un programa de estudios, un grupo de investigación, una revista académica, un departamento académico o en el mejor de los casos, una facultad o escuela? ¿Serán algo tan novedoso que todavía no han sido creados para el caso de Perú? ¿O será que, tratándose de una minoría (comunidad pequeña) dentro de otra minoría (los latinoamericanos judíos), nunca se crearán Estudios Judíos en el

Perú?

En lo que sigue, se intentará dar cuenta de la situación de aquello que, caritativamente, podríamos denominar "Estudios Judíos" sobre y desde el Perú, entendidos como la aproximación al encuentro entre "lo judío" y "lo peruano". Debido al reducido número de trabajos sobre este tema que califican como académicos, me tomaré la libertad de incluir en la discusión trabajos que no son académicos pero han tenido más impacto que los anteriores. En primer lugar y con el objeto de ofrecer un panorama general, se hará un breve recuento de otros trabajos elaborados en Perú que se han ocupado de minorías de origen extranjero, el campo en el que se han insertado con mayor comodidad-aunque no 
de forma exclusiva- los trabajos sobre "lo judío" y "lo peruano" producidos en el Perú hasta el momento. En segundo lugar, se expondrán brevemente los temas abordados en los trabajos de mayor relevancia del supuesto campo de los "Estudios Judíos" en el Perú, para finalmente, presentar una breve reflexión.

\section{Pensar lo peruano y lo judío}

En sentido estricto, no ha surgido en Perú una disciplina, una escuela ni una línea de investigación que aborde lo peruano y lo judío, a diferencia de lo que parece haber ocurrido en otros países de la región, especialmente en aquellos en los que la población judía es numéricamente mayor (Argentina, Brasil o México) o en los que las posiciones políticas de los países frente al Estado de Israel han desatado controversia a nivel internacional (Cuba o más recientemente, Venezuela).

Esta ausencia de Estudios Peruano-Judíos se explica no solamente porque en Perú la población judía en la actualidad es reducida (un poco menor a 2,000 judíos, según DellaPergola (2013) ni por la relación que mantiene dicho país con Israel. Se debe también a que dentro del esfuerzo mayor por institucionalizar los Estudios Judíos en general, las comunidades judías más pequeñas de América Latina (como la de Perú) parecen tener menos qué decir sobre lo latinoamericano judío en comparación con las comunidades de mayor tamaño.

Adicionalmente, el Perú es un país en el que la actividad académica ha estado significativamente restringida a la docencia y en la que se ha hecho poca investigación en general ${ }^{1}$. En otras palabas: si hasta hace no mucho era necesario, por ejemplo, justificar la existencia de programas académicos sobre los judíos estadounidenses destacando para

1 La universidad peruana que ocupa el puesto más elevado en el ránking QS World Universities aparece entre los puestos 491-500 del mundo y en la categoría "research" sólo obtuvo la calificación "médium". Véase la página de QS Top Universities, disponible en http://www.topuniversities.com/. 
ello su aporte al entendimiento del mundo occidental cristiano (Heschel, 1998); y si, con mucho esfuerzo, se están conformando grupos de investigación y proyectos de mayor escala en países que lideran la actividad académica en general en América Latina; ¿cómo es posible pensar en Estudios Judíos en un país que ha quedado relegado en producción académica en general?

De forma paralela, la inexistencia de Estudios Judíos del Perú o Estudios Judeo-Peruanos tiene que ver también con el estado de los Estudios Étnicos o estudios sobre las minorías peruanas de origen extranjero y otras minorías que, en términos de interés académico y ante la precariedad de la actividad de investigación en el país, parecen haber sido descuidadas en favor del relato "fundacional" de lo peruano hasta hace no mucho, a saber, el encuentro entre indígenas y europeos provenientes de la península ibérica.

Entonces, es poco lo que se ha producido en el Perú sobre lo judío y lo peruano y la mayoría de productos no son académicos. Esto, para bien y para mal, no solo es característico del estudio sobre lo judío y lo peruano. También los estudios sobre otras minorías peruanas de origen extranjero, como propongo a continuación, han atravesado un camino similar.

\section{Minorías peruanas de origen extranjero}

En el proceso por entender la inserción social de los judíos en Lima para la elaboración de mi investigación doctoral, tuve que revisar trabajos sobre otros grupos minoritarios que pueden trazar su presencia en el país a la inmigración en el período republicano. Estos trabajos han aparecido por lo menos desde la década de 1970 en el marco de una línea de investigación creada por los Departamentos de Antropología de la Universidad Nacional Mayor de San Marcos y la Pontificia Universidad Católica del Perú (Millones, 1973), con el propósito de estudiar "el prejuicio racial en el Perú" (p. 13). Una primera publicación en este contexto sobre los inmigrantes de origen africano y sobre los de 
origen chino exploraba la situación de estos inmigrantes en la Colonia y solo para el caso de la minoría china el estudio se extendía hasta el siglo $\mathrm{XX}$.

No es casual que este interés por estudiar a las minorías de origen extranjero haya surgido en la década de 1970, con el inicio de una serie de transformaciones sociales desatadas a partir del gobierno dictatorial de izquierda del General Juan Velasco Alvarado (19681975). Uno de los hitos de este gobierno fue la promulgación e implementación de la Ley de Reforma Agraria, que, junto a otras reformas, reforzaría un proceso que condujo al “ocaso de la oligarquía” peruana (Cotler, 2005; Flores Galindo 1986/2013). Este golpe al régimen oligárquico no significó una plena reparación de las injusticias que el Perú republicano heredara desde la Colonia (Cotler, 2005). No obstante, supuso un cambio en la composición de las clases dominantes del país, abriendo sus puertas a nuevos actores, algunos de los cuales habían sido anteriormente marginados por no descender del grupo adecuado y otros, por no tener abuelos enterrados en el país. No obstante y quizás de forma paralela a los intentos por enfrentar la desigualdad en el país, este esfuerzo por estudiar a las minorías de origen extranjero parece haber quedado frustrado luego de sus primeros frutos. Dentro de las Ciencias Sociales en general, han tenido mayor presencia los estudios andinos (incluyendo los estudios sobre la violencia política ocasionada por el terrorismo), los trabajos en temas de desarrollo y desigualdad, los estudios socioambientales y los estudios que analizan los conflictos sociales que han afectado al país, por nombrar solo algunos ${ }^{2}$.

Las transformaciones sociales vinculadas con los cambios políticos de 1968 se hicieron evidentes en la década de 1990, en la que hubo también un incremento en publicaciones sobre minorías de origen extranjero, a propósito del ingreso de peruanos pertenecientes a algunas de estas minorías al ámbito de la política. Éstos adquirieron una visibilidad sin

2 Véanse como muestra los catálogos del Instituto de Estudios Peruanos (IEP) y del Fondo Editorial de la Pontificia Universidad Católica del Perú (PUCP). Disponibles en http://www.iep.org.pe/catalogo_fe.html y http://www.fondoeditorial.pucp.edu.pe/. 
precedentes tras la elección de un Presidente peruano de primera generación (de ascendencia japonesa) en las elecciones presidenciales de 1990. En palabras de una autora peruano-árabe,

la victoria de Alberto Fujimori en las elecciones presidenciales de 1990 en el Perú tuvo un fuerte impacto en la población peruana de origen extranjero y empujó a muchos intelectuales a repensar la construcción de la identidad en un país donde el problema de la inmigración había sido relativamente olvidado (Bartet, 2005, p. 175).

Fuera del ámbito académico, llaman la atención una nueva serie de trabajos realizados en Perú sobre las minorías peruanas de origen extranjero. Algunos se han enfocado en aquellas minorías que, por tamaño, se han hecho más visibles en las ciudades más pobladas del país y que hacia el final del siglo XX e inicios del XXI, son representativas en el sector privado, en la sociedad civil y en el Estado (Morimoto 1999; Bonfiglio, 2001; Derpich, 1999). En líneas generales, estos trabajos - y aquellos que se ocupan también de grupos menos numerosos y menos visibles-, destacan el factor diaspórico o "excepcional" (Lesser y Rein, 2006) de dichas minorías, aunque hay trabajos como los de Morimoto (1991 y 1999) sobre los nikkei que hacen énfasis en las estrategias de enraizamiento, que podríamos también llamar "el proceso de peruanización". Algunos de estos trabajos han sido editados y publicados por el Fondo Editorial del Congreso de la República del Perú3 ${ }^{3}$

Acerca del caso de la inmigración asiática en el Perú, destacan los trabajos elaborados por Amelia Morimoto (1991 y 1999) para el caso específico de los inmigrantes provenientes del Japón y sus descendientes y una serie de trabajos que se ocupan de los inmigrantes provenientes de China y sus descendientes (Chuhue, Jing Na y Coello, 2012; Derpich, 1999, entre otros). Ambos casos, inmigrantes chinos y japoneses, son de gran

3 Véase el catálogo del Fondo Editorial del Congreso de la República, disponible en http://www.congreso.gob.pe/FondoEditorial/?K=578. 
relevancia porque hacia la década de 1930 conformaban los dos grupos étnicos más numerosos en el Perú que, a diferencia de otras minorías migrantes del mismo período, accedieron al país durante la segunda mitad del siglo XIX de forma organizada, a través de contratos y con el propósito explícito de conseguir mano de obra para las haciendas (Yamawaki, 2002). Los judíos, los árabes y los europeos no españoles, en cambio, no llegaron al país de manera organizada a través del Estado.

Entre las minorías de ascendencia extranjera con mayor visibilidad se encuentran los peruanos descendientes de italianos que llegaron al país en el período republicano. Se trata del grupo inmigrante más numeroso proveniente de Europa -cerca de 12,000 (Bonfiglio, 2001)- para el período que va de la segunda mitad del siglo XIX a la primera del siglo XX. El autor de forma expresa propone "(el) estudio de la etnicidad de los inmigrantes italianos (...) en estos tiempos de resurgimiento de las etnicidades y de aceptación de la multietnicidad" (p. 334). En otras palabras, el final del siglo XX se presenta como el momento propicio para atender la diferenciación de las minorías porque es una marca de orgullo y distinción, especialmente si uno descubre, como en el caso de los inmigrantes europeos al Perú, que hay una correlación entre una "alta valoración étnica" (Bonfiglio, 2001, p. 327) y "un ascenso automático en la jerarquía social peruana" (p. 327).

Un aspecto curioso acerca de la manera en que han sido abordados estos trabajos no académicos sobre minorías de origen extranjero es que desde la mirada excepcionalista, expresan una suerte de denuncia de las prácticas post-coloniales peruanas halladas por los inmigrantes del período republicano. Por ejemplo, algunos autores refuerzan la idea de que el ascenso social de las minorías de origen extranjero en el Perú se debe al trabajo arduo y el valor del ahorro entre inmigrantes asiáticos y europeos no españoles, en oposición a una suerte de cultura "peruana" del ocio hallada por dichos inmigrantes al arribar al país (Yamawaki, 2002; Bonfiglio, 2001). 
No obstante, el ascenso social no ocurrió en simultáneo para todas las minorías de origen extranjero, y en ese sentido, los europeos no españoles (especialmente los italianos, que eran mayoría) habrían experimentado una suerte de privilegio. Al respecto, Giovanni Bonfiglio (2001) sostiene que "los descendientes de italianos engrosaron las capas medias y medias altas de la sociedad peruana y estuvieron en la vanguardia de los procesos económico y sociales que actuaron en [el] siglo [XX]” (p. 319). Dado que los descendientes de italianos, como otros europeos no españoles en el Perú, se insertaron económicamente a través de la industrialización del país, Durand (1988) afirma que esta inserción “'democratizó y diversificó la estructura de poder oligárquico” (p. 28), algo que se pone de manifiesto especialmente con el gobierno de Juan Velasco Alvarado, cuando al resquebrajarse dicho poder, los industriales peruanos, entre ellos algunos de ascendencia italiana, aparecen como una suerte de reemplazo de la clase dominante.

Es pertinente señalar aquí que al menos en el marco de la producción no académica sobre las minorías de origen extranjero, los judíos han aparecido curiosamente como parte de las inmigraciones europeas no españolas. Muestra de ello es una versión resumida del trabajo de dos dirigentes comunitarios de las últimas décadas del siglo $\mathrm{XX}$, León Trahtemberg (el Supervisor Pedagógico del colegio judío de Lima) en co-autoría con Eduardo Bigio (quien presidiera el Comité de Relaciones Humanas de la Asociación Judía del Perú por tres décadas). Dicho texto fue elaborado especialmente para un compendio editado por el Fondo Editorial del Congreso del Perú bajo el título La presencia europea en el Perú (Bonfilgio, 2001). Así, dicho compendio contiene un capítulo sobre la inmigración judía en el país, lo que resulta de interés porque dicho capítulo incluye detalles sobre la presencia sefaradí, que para el caso peruano proviene de Marruecos (especialmente a la selva) y del desintegrado Imperio Otomano. Esta confusa inclusión revela, por un lado, que "ser europeo" algunas veces no depende del lugar de origen, sino del arbitrario estatus alcanzado en las Américas. Por otro lado, inclusión de los peruanos judíos como parte del conjunto de europeos no españoles en el Perú refuerza la idea de que la afiliación religiosa no es una traba para encontrar elementos de 
cohesión, especialmente luego del giro de la Iglesia Católica hacia la mejora en las relaciones con religiones no cristianas, tal como se declara en Nostra Aetate (1965).

Con esta breve revisión se han querido señalar tres cosas. En primer lugar, que el interés por estudiar a las minorías peruanas de ascendencia extranjera es relativamente reciente. En segundo lugar, que está asociado a las transformaciones sociales de las últimas tres décadas del siglo XX en el Perú, que significaron el ingreso a las clases dominantes de nuevos actores. En tercer lugar, que ha tenido mayor acogida fuera del ámbito académico. En este marco, ¿qué ha ocurrido con el discurso sobre lo peruano y judío elaborado desde el Perú?

\section{Hecho en Perú: productos sobre lo judío realizados en Perú}

Con el propósito de evaluar la situación de la producción académica respecto del cruce entre lo peruano y lo judío, ser realizó una revisión de los catálogos virtuales de las bibliotecas más importantes del país, a saber, los de la Biblioteca Nacional del Perú, la Pontificia Universidad Católica del Perú, la Universidad Nacional Mayor de San Marcos, la Universidad de Lima, la Universidad Peruana Cayetano Heredia, la Universidad del Pacífico y la Universidad Antonio Ruiz de Montoya. Fueron encontrados al menos once trabajos académicos y menos de una veintena trabajos no académicos que podrían mantener relación con el tema discutido aquí.

En líneas generales, los escasos trabajos realizados en el Perú que versan sobre algo peruano y algo judío se concentran en dos temas específicos: para el caso de los académicos y en términos generales, los conversos (judíos de la península ibérica convertidos al cristianismo) en el Perú colonial; y para el caso de los productos no académicos, la presencia judía en el Perú a partir del período republicano. Esta última ha sido entendida, notablemente, como (1) algo aislado o separado del resto de la sociedad; (2) desde la sensación de amenaza o exclusión sufrida por los judíos frente a la sociedad 
anfitriona y/o 3) desde una perspectiva "excepcionalista" según la cual se asumen rasgos diaspóricos compartidos por los judíos en tanto grupo, sin prestar atención a la experiencia nacional (Lesser y Rein, 2006). El estudio de lo judío y peruano ha sido aún menos explorado desde la perspectiva de los estudios de religión, motivo por el cual, considero pertinente ubicarlos dentro de los estudios sobre las minorías peruanas de origen extranjero. En ese sentido, el interés de diversos autores por la inmigración judía del período republicano y por los elementos que, en principio, la mantendrían diferenciada del resto de la sociedad (como algo exótico), es compartido por los autores de los también raros trabajos que se han realizado desde el Perú sobre minorías peruanas de origen extranjero.

\section{Los trabajos académicos}

Concretamente, entre los trabajos académicos hallados, cinco pertenecen a la especialidad de Historia del Perú colonial; tres califican como trabajos de Historia de la inmigración al Perú en el período republicano; uno es un trabajo de Historia de la prensa peruana del siglo XIX, uno pertenece al campo de la Antropología de las Religiones en el Perú y uno pertenece al ámbito de los Estudios Sociales sobre Minorías peruanas. Ninguno de estos trabajos ha conseguido ser algo más que la satisfacción de una curiosidad circunstancial hasta el momento. De hecho, los trabajos que, en términos relativos, más eco han hecho sobre lo judío y lo peruano, escapan del ámbito académico, de forma paralela a lo que parece haber ocurrido con los estudios sobre otras minorías de origen extranjero.

Al buscar trabajos académicos que se ocupen de lo peruano y lo judío se registró sólo uno elaborado antes del que constituye un hito para los estudios sobre minorías étnicas en el país: el gobierno dictatorial de izquierda del General Juan Velasco Alvarado (19681975). Este trabajo es una tesina de la especialidad de Historia de 1940 en la que el autor "denuncia" la presencia judía y japonesa en el Perú como un problema para el país, inspirado en El judío internacional de Henry Ford o incluso Mein Kampf. Fuera de ese 
singular caso, se encontraron nueve trabajos elaborados posteriormente al golpe militar y la promulgación de la Ley de Reforma Agraria de Velasco Alvarado, coincidiendo con lo expuesto anteriormente para el caso de otras minorías de origen extranjero.

La única reacción conocida a la tesina de 1940 ha sido una tesis de Licenciatura en Historia en la Pontificia Universidad Católica del Perú presentada por Sonia Fleischman en 1985, en la que examinaba la inmigración judía al Perú en un intento por demostrar que la presencia judía en el país había rendido frutos, esto es, que hay valiosos aportes ligados a ella. Este es otro caso de un trabajo académico que surge como intento por reivindicar a una minoría frente a antiguos detractores, enfocándose en lo que la hace "excepcional", en instancias de exclusión y rechazo y en los logros que dicha minoría consiguió a pesar de ello. Hasta donde tengo conocimiento, este trabajo no derivó en publicaciones posteriores.

Otro trabajo que aborda la inmigración judía al Perú fue publicado en una revista de la Universidad Nacional Mayor de San Marcos en 2010, apoyándose en un puñado de entrevistas que una vez más, priorizan lo singular de lo judío ante la experiencia de enraizamiento o incluso, ante la comparación con otras minorías.

Del resto de trabajos académicos encontrados sobre lo judío y el Perú, destacan los que abordan el tema de los conversos, descritos como judíos portugueses convertidos al cristianismo que arribaron al Virreinato del Perú, en el que operó por 250 años una sucursal de la Inquisición española (Museo del Congreso y de la Inquisición, 2016).

Adicionalmente, se registran dos trabajos inusuales que, a diferencia de los anteriores, presentan lo judío de forma comparada con otro "otro": en un caso, se trata de una comparación sobre literatura anti-judía y anti-africana y su papel en la prensa peruana decimonónica y en el otro, de una comparación del capital religioso de peruanos judíos y musulmanes. Una vez más, se trata de trabajos que no están pensados como parte de un proyecto mayor de investigación ni como parte de una discusión más amplia. 
Finalmente, mi tesis de Doctorado en Sociología aborda los cambios atravesados por la comunidad organizada de Lima durante sus primeros 70 años (1944-2014), tomando como punto de partida la creación de una organización paraguas. El trabajo combina una mirada proveniente de la Sociología de la Religión con la de los Estudios Étnicos para examinar la versión limeña de "lo judío", en virtud de las relaciones entre judíos y no judíos peruanos en la esfera privada y en la pública, algo que no ha sido estudiado anteriormente. Un primer producto de esta investigación es un capítulo titulado Oferta religiosa judía en la ciudad de Lima, que apareció en 2016 en el libro multidisciplinario Diversidad religiosa en el Perú. Miradas múltiples y que reúne contribuciones acerca del catolicismo, el cristianismo evangélico y el judaísmo. Considero relevante señalar que el libro ha sido co-editado por el Fondo Editorial de la Pontificia Universidad Católica del Perú y el Centro de Estudios y Publicaciones, éste último un proyecto editorial especializado en temas de teología cristiana, religión, derechos humanos, entre otros. Hasta donde tengo conocimiento, es la primera vez que se incluye un capítulo sobre judaísmo en un libro sobre religiones en el Perú.

\section{Los trabajos no académicos}

Quizás como muestra de la poca institucionalización en general de la actividad de investigación académica en el Perú hasta hace no mucho, los trabajos que no pertenecen al ámbito académico han tenido más impacto que aquellos que califican como propiamente académicos. De hecho, al examinar la producción sobre lo judío y lo peruano es casi ineludible referirse a la serie preparada por León Trahtemberg (1987, 1988, 1989 y 1991), que se aproximó al tema desde la perspectiva de quien ocupa una función dentro de la organización comunitaria y se dirige hacia una audiencia netamente judía. Aún más, el motivo detrás de los trabajos es, según el autor, preservar la memoria del "pueblo judío" ante la potencial amenaza de desaparición de comunidades judías esta vez a propósito de nuevos movimientos migratorios y del siempre temido "proceso 
asimilatorio" (Trahtemberg, 1989, p.5). Si bien algunos de sus trabajos fueron elaborados en el marco de sus estudios de Maestría en Educación en la Universidad Hebrea de Jerusalem, fueron publicados por las distintas congregaciones de la Asociación Judía del Perú y en ese sentido, vendrían a ser el discurso "oficial" de la comunidad judía de Lima desde el final de la década de 1980.

Los trabajos de Trahtemberg proponen una aproximación a la inmigración de judíos al Perú similar a la realizada por los autores mencionados líneas arriba para los casos de otros grupos inmigrantes que no llegaron de forma organizada: primero se hace un listado de nombres de inmigrantes notoriamente judíos y en algunos casos, se describe la actividad a la que se dedicaron al arribar al país, con el objetivo ulterior de demostrar la contribución del grupo minoritario a la sociedad en general. Luego, se confecciona una descripción de las asociaciones y organizaciones creadas como muestra del dinamismo del grupo minoritario, destacando los eventos de rechazo o exclusión (Trahtemberg 1987, 1989). En ellos no se problematizan otros aspectos relativos al enraizamiento, como las relaciones entre peruanos judíos y no judíos o la situación de otras minorías en el país.

Adicionalmente, ha aparecido un libro editado por el Fondo Editorial del Congreso del Perú que exhibe, utilizando fotografías de archivos de la Biblioteca Nacional del Perú y de archivos privados, un "retrato de la comunidad judio-peruana" (Salcedo y Mitrani, 2002). Dicha publicación había sido elaborada en la década de 1980 pero por falta de fondos e interés, no fue publicada sino hasta el gobierno de Alejandro Toledo (20012006), cuya esposa y algunos allegados fueron judíos. La presentación del libro fue motivo para la organización de un conversatorio sobre la presencia judía en el Perú, que derivó en una segunda publicación en 2006 que, al igual que otras publicaciones editadas por el Congreso del Perú, prioriza lo exótico y distinto en una compilación de relatos sobre inmigrantes, testimonios de judíos prominentes en el Perú, recetas de comida, entre otros (Mitrani, 2006). No obstante, es interesante destacar que ambas publicaciones fueron preparadas por descendientes de judíos que no han formado parte de la 146

Cuadernos Judaicos ISSN: 0718-8749

n 34-diciembre 2017 
organización comunitaria de Lima pero que han mantenido contacto con parientes y conocidos que sí han sido parte de ella.

La Shoá ha sido un tema que ha generado también interés en las últimas décadas fuera del marco académico peruano. Además de testimonios de sobrevivientes que llegaron al Perú y que publicaron sus memorias de forma privada, ha aparecido un libro del género de periodismo de investigación aplicado a la Historia (Coya, 2010), acerca de la experiencia de ciudadanos peruanos en la Shoá (es decir, peruanos judíos que migraron a Europa en el período de la guerra).

A esto se suma un libro que recoge testimonios sobre las migraciones producidas por la situación desfavorable de los judíos de Europa Central y Occidental durante la primera mitad del siglo XX, publicado en 2012 por una de las congregaciones religiosas de la Asociación Judía del Perú (la organización paraguas comunitaria oficial para los judíos del país) y dirigido, como todas las publicaciones comunitarias, a una audiencia interna.

Para el caso de la política y los judíos en Perú, el registro evidencia un artículo y un libro que abordan el involucramiento de judíos en la actividad política de la izquierda en el Perú. El libro llama la atención por haber sido encargado y publicado por la iniciativa de un conocido empresario judío que ha formado parte de los círculos de la política peruana durante décadas y que ha ocupado brevemente un importante cargo ministerial en el gobierno de Ollanta Humala (2011-2016). En ambos casos y debido al período en que se enmarca el trabajo (la primera mitad del siglo XX), el foco está en las experiencias y perspectivas de los inmigrantes judíos oriundos de Europa (de donde provenía dicha orientación política) y en la primera generación de peruanos judíos ashkenazíes, dejando fuera a los sefaradíes y de cierto modo, a las generaciones siguientes de peruanos judíos ${ }^{4}$.

4 Véase Gonzáles (2014). 
Finalmente, también en el terreno de trabajos de divulgación hay un par de trabajos acerca de los judíos portugueses en la Colonia, lo que parece confirmar lo señalado líneas arriba, es decir, que el interés por el encuentro entre lo peruano y lo judío se ha dado, hasta ahora, bajo dos temas de interés (conversos en la Colonia e inmigración judía del período republicano), independientemente de la naturaleza -académica o de divulgaciónde los productos.

\section{A modo de conclusión}

La pregunta acerca de la situación de los Estudios Judíos desde el Perú debe estar ligada a la pregunta acerca de la institucionalización de los Estudios sobre Etnicidad, los Estudios sobre Religiones y los Estudios sobre Minorías en el país, lo que podría tener sentido también $\multimap$ no- para otros países de la región.

Luego de una revisión de la producción académica sobre lo peruano y lo judío realizada en el Perú, se puede considerar pertinente afirmar que lo poco que se ha producido, dialoga en líneas generales con dos campos, a saber, los estudios históricos sobre la Colonia en el Perú, y los estudios sobre minorías de origen extranjero del período republicano. La aparición de trabajos que no son académicos (esto es, que no han sido editados ni publicados en el marco del mundo académico, no han atravesado procesos de revisión ni evaluación siguiendo las reglas de dicho ámbito) acerca de las minorías de origen extranjero, parece indicar que al menos el campo de los estudios sobre minorías de origen extranjero todavía se encuentra en proceso de consolidación y que antes que un interés propiamente académico, ha surgido independientemente de la actividad académica, como una reacción al ingreso al espacio público peruano de actores pertenecientes a dichas minorías.

De ahí que no se hayan creado líneas de investigación, programas académicos ni escuelas que propongan métodos de aproximación ni un objeto específico de estudio. Mi propio 
intento por estudiar lo peruano y lo judío puede ser una muestra de lo que ello significa. Tuve que inscribir mi tesis de Doctorado en Sociología en la línea de investigación sobre Religión y Sociedad y de forma paralela, en el Seminario Interdisciplinario de Estudios Religiosos (SIER) de la Pontificia Universidad Católica del Perú. Hasta donde tengo conocimiento, el mío es el primer trabajo que dialoga directamente con el discurso académico sobre religiones en el Perú y hasta la fecha, es poco probable que surjan oportunidades de continuar investigando sobre lo judío y lo peruano en ese marco. No obstante, es de esperarse que paulatinamente crezca interés en este campo a la luz de los cambios en la composición religiosa del país que, como en otras partes de la región, ha ido migrando de la Iglesia Católica hacia las iglesias reformadas (Pew Research Center, 2014).

Adicionalmente, el caso de los peruanos judíos podría eventualmente dialogar no solo con los estudios sobre minorías sino específicamente con los estudios sobre la comunidad pequeña latinoamericana -judía o no- en ciudades grandes, como es el caso de Lima. Porque una cosa es ser minoría en un país, y otra, es ser minoría dentro de la minoría. Es decir, así como no es lo mismo estudiar a las iglesias reformadas en Brasil o Guatemala que hacerlo en Uruguay; tampoco lo es estudiar a los peruanos o ecuatorianos judíos que a los argentinos o brasileños judíos. Así, por ejemplo, he encontrado que, en una comunidad pequeña, como la peruana judía en Lima, lo religioso y lo étnico-cultural tienden a mezclarse, en buena medida porque al no contar con suficientes personas que compartan el mismo origen se hace más difícil preservar la diversidad étnica (como la lengua o las costumbres). Además, la poca visibilidad de las comunidades pequeñas refuerza la sensación de "exotismo", ya no solamente porque los peruanos judíos representan el $0.006 \%$ de la población nacional ${ }^{5}$ sino porque es exótico ser peruano dentro del mundo judío.

5 Según el Instituto Nacional de Estadística e Informática (INEI), en 2014 la población nacional asciende a 30’814,175 habitantes. Véase INEI, 2014. 
De ese modo, podría analizarse el peso y la relevancia que tienen, por ejemplo, los centros comunitarios (judíos, japoneses, árabes, chinos, gitanos, evangélicos, musulmanes, etc.) para las comunidades pequeñas en comparación al que tienen en el caso de comunidades grandes en virtud de la formación de identidades o de la preservación de la diferenciación social. También podría estudiarse cómo incide en la institucionalización de los Estudios Judíos la manera en la que lo judío (o lo minoritario, en América Latina), en tanto cultura religiosa y etnicidad, se integra al país en cuestión, independientemente de la manera en que los individuos se integren a la ciudad. Y en definitiva, habría que rastrear el surgimiento de estudios sobre minorías en América Latina para comparar el auge que han tenido en algunas partes de la región frente a otras en las que queda pendiente recuperar las perspectivas de quienes aparecen como minoría entre las minorías. 


\section{Bibliografía}

Bartet, L. (2005). Memorias de cedro y olivo. Lima: Fondo Editorial del Congreso del Perú.

Bonfiglio, G. (2001). La presencia europea en el Perú. Lima: Fondo Editorial del Congreso de la República.

Cotler, J. (2005). Clases, estado y nación en el Perú. Lima: Instituto de Estudios Peruanos.

DellaPergola, S. (2013). World Jewish Population, 2013. En Dashefsky, A. y Sheskin, I. (eds.), The American Jewish Yearbook, 2013, vol. 113 (pp. 279-358). Dordrecht: Springer.

Derpich, W. (1999). El otro lado azul: 150 años de inmigración china al Perú. Lima: Fondo Editorial del Congreso de la República.

Durand, F. (1988). La burguesía peruana: los primeros industriales, Alan García y los empresarios. Lima: DESCO.

Flores Galindo, A. (1986/2013). La tradición autoritaria. Violencia y democracia en el Perú. En Portocarrero, G. (ed.), Sombras coloniales y globalización en el Perú de hoy (pp. 19-55). Lima: Red para el Desarrollo de las Ciencias Sociales en el Perú.

Gonzáles, O. (2014). La presencia judía en la izquierda peruana. Lima: Otra Mirada. 
Heschel, S. (1998). Jewish Studies as Counterhistory. En Biale, Galchinsky y Heschel (1998). Indiser/Outsider. American Jews and Multiculturalism (pp. 101-115). Berkeley y LA: University of California Press.

Lesser, J. y Rein, R. (2006). Challenging particularity. Jews as a lens on Latin American ethnicity. Latin American and Caribbean Ethnic Studies 1 (2), setiembre, 249-263.

Lindstrom, N. (2001). Recent tendencies in Latin American Jewish Studies. Shofar: an interdisciplinary journal of Jewish Studies 19 (3), 23-32.

Mendes-Flohr, P. y J. Reinharz (1995). The Jew in the Modern World. New York: Oxford University Press.

Millones, L. (1973). Minorías étnicas en el Perú. Lima: Pontificia Universidad Católica del Perú.

Mitrani, H. (2006). Ser judío en el Perú. Lima: Fondo Editorial del Congreso del Perú.

Morimoto, A. (1991). Población de origen japonés en el Perú: perfil actual. Lima:

Centro Cultural Peruano-Japonés.

Morimoto, A. (1999). Los japoneses y sus descendientes en el Perú. Lima: Congreso de la República del Perú.

Pew Research Center (2014). Religion in Latin America: Widespread Change in a 
Historically Catholic Region. Disponible en

http://www.pewforum.org/files/2014/11/Religion-in-Latin-America-11-12-PM-full-

PDF.pdf

Romero, C. (comp.) (2016). Diversidad religiosa en el Perú. Miradas múltiples. Lima:

PUCP; CEP.

Salcedo, L. y H. Mitrani. (2002). El eterno retorno: retrato de la comunidad judioperuana. Lima: Fondo Editorial del Congreso del Perú.

Trahtemberg, L. (1987). La inmigración judía al Perú 1848-1948. Lima: Asociación de Beneficencia y Culto 1870.

Trahtemberg, L. (1988). Demografía judía del Perú. Lima: Unión Mundial ORT.

Trahtemberg, L. (1989). Los judíos de Lima y las provincias del Perú. Lima: Unión Israelita del Perú.

Trahtemberg, L. (1991). Participación del Perú en la Partición de Palestina. Lima: Colegio León Pinelo.

Yamawaki, C. (2002). Estrategias de vida de los inmigrantes asiáticos en el Perú. Lima: IEP; JCAS.

\section{Fuentes web}


Catálogo del Fondo Editorial del Congreso de la República. http://www.congreso.gob.pe/FondoEditorial/?K=578.

Catálogo del Fondo Editorial de la Pontificia Universidad Católica del Perú. Disponible en http://www.fondoeditorial.pucp.edu.pe/.

Catálogo virtual de la Biblioteca de la Universidad Antonio Ruiz de Montoya. Disponible en http://biblio.uarm.edu.pe/.

Catálogo virtual del sistema de bibliotecas de la Pontificia Universidad Católica del Perú. Disponible en http://biblioteca.pucp.edu.pe/.

Catálogo virtual de la Biblioteca de la Universidad del Pacífico. Disponible en https://campusvirtual.up.edu.pe/Biblioteca/default.aspx?utm_source=CampusVirtual\&ut $\underline{\text { m_medium=banner\&utm_campaign=Biblioteca. }}$

Catálogo virtual de la Biblioteca de la Universidad de Lima. Disponible en http://www.ulima.edu.pe/departamento/biblioteca.

Catálogo virtual del sistema de bibliotecas de la Universidad Nacional Mayor de San Marcos. Disponible en http://sisbib.unmsm.edu.pe/.

Catálogo virtual del sistema de bibliotecas de la Universidad Peruana Cayetano Heredia. Disponible en http://dugic.cayetano.edu.pe/.

Catálogo del Instituto de Estudios Peruanos. Disponible en http://www.iep.org.pe/catalogo_fe.html 
Página Web del Museo del Congreso y de la Inquisición. Disponible en http://www4.congreso.gob.pe/museo/index.html

Página Web de QS Top Universities. Disponible en http://www.topuniversities.com/. 